\title{
The effect of simvastatin on the regeneration of surgical cavities in the femurs of rabbits ${ }^{1}$
}

\author{
José Eduardo Gomes Cardim Rosselli', Dulce Maria Fonseca Soares Martins" ${ }^{\mathrm{II}}$, José Luiz Martins ${ }^{\mathrm{III}}$, Claudia Regina Gomes \\ Cardim Mendes de Oliveira ${ }^{\mathrm{IV}}$, Djalma José Fagundesv', Murched Omar Tahav
}

${ }^{\mathrm{I}}$ Fellow PhD degree, Postgraduate Program in Surgical Science Interdisciplinary, Department of Surgery, Sao Paulo Federal University (UNIFESP), Sao Paulo-SP, Brazil. Conception, design and scientific content of the study; technical procedures; analysis and interpretation of data; manuscript writing. IIPhD, Associate Professor, Department of Surgery, Division of Plastic Surgery, UNIFESP, Sao Paulo-SP, Brazil. Scientific content of the study, critical revision.

IIIPhD, Full Professor, Pediatric Surgery Division, Department of Surgery, UNIFESP, Sao Paulo-SP, Brazil. Manuscript writing, critical revision.

${ }^{\text {IV }} \mathrm{PhD}$, Department of Pathology, Institute of Orthopedics and Traumatology, School of Medicine, Sao Paulo University (FMUSP), Sao Paulo-SP, Brazil. Histological and immunohistochemical analysis.

${ }^{\mathrm{v}} \mathrm{PhD}$, Associate Professor, Operative Technique and Experimental Surgery Division, Department of Surgery, UNIFESP, Sao Paulo-SP, Brazil. Critical revision.

\begin{abstract}
PURPOSE: To evaluate the effect of a local application of simvastatin gel in repairing bone defects in the femurs of rabbits.

METHODS: Two standard surgical cavities were created in the femoral epiphysis of 18 rabbits. In the simvastatin group (SG), the cavities were filled with a collagen sponge soaked in $0.5 \mathrm{ml}$ of a simvastatin $(1 \mathrm{mg})$ gel, and the cavities were covered with a biological membrane. The bone cavities in the second group (control group) were filled with a blood clot and covered with a biological membrane. On the $7^{\text {th } 21 \text { st }}$ and $42^{\text {nd }}$ days, six animals in each group were euthanized, and the femurs were subject to histological evaluation (vascularity, fibrosis, reactive bone formation, osteoblasts, and osteoclasts) and immunohistochemical (anti-VEGF and anti-osteocalcin) analysis. The results were analyzed using a Wilcoxon test $(\mathrm{p}<0.05)$.

RESULTS: There were significant differences between the two groups: the SG had greater scores in comparison with the CG in terms of the degree of vascularity on the $7 \mathrm{t}^{\mathrm{h}}$ and the 21 st days, fibrosis on the $21^{\text {st }}$ day, bone formation reaction on the $21^{\text {st }}$ and the $42^{\text {nd }}$ days and the number of osteoblasts and osteoclasts on the $42^{\text {nd }}$ day. The immunohistochemical expression was also greater for osteocalcin and VEGF on the $7^{\text {th, } 21 \text { st }}$ and $42^{\text {nd }}$ days.
\end{abstract}

CONCLUSION: Surgical defects created in rabbit femurs were treated locally with simvastatin gel to stimulate bone repair, which promoted an ameliorative effect in the morphological and immunohistochemical markers of bone regeneration.

Key words: Simvastatin. Bone Regeneration. Bone Morphogenetic Proteins. Rabbits. 


\section{Introduction}

Experimental models of bone regeneration using critical defects with no spontaneous regeneration have been used to analyze different substances with osteoinductive properties, which are specifically able to induce the differentiation of osteoprogenitor cells into osteoblasts and induce bone formation ${ }^{1,2}$. New bone formation is triggered by an inducing substance present in the bone collagen matrix known as bone morphogenetic protein (BMP) $)^{3}$.

BMPs are non-collagenous proteins responsible for chemotaxis, proliferation of mesenchymal cells, osteoblast differentiation and bone formation ${ }^{4,5}$. Drugs called statins have been used for over 30 years to treat hyperlipidemia and arteriosclerosis. These drugs may stimulate the expression of BMP2 and prevent the prenylation of some GTPase proteins ${ }^{6,7}$. Statins may also stimulate the expression of vascular endothelial growth factor (VEGF), which stimulates the differentiation of endothelial progenitor cells (EPCs) and, thus, stimulate angiogenesis ${ }^{8}$. Statins also reduce bone resorption and therefore inhibit farnesylpyrophosphate (geranilgeranilpirofosfato), which is essential for cell differentiation and the function of osteoclasts ${ }^{6}$. However, clinical studies and systematic reviews have shown no correlation between the use of oral statins and bone density, osteoporosis or the prevention of bone fractures ${ }^{9,10}$. In vitro studies of simvastatin and its local application in experimental bone defects has demonstrated, after histological and immunohistochemical analyses, that simvastatin increases the expression of the growth factors VEGF and BMP2, which are major regulators of angiogenesis and osteogenesis ${ }^{11,12}$.

A consensus has not yet been reached on the dose of simvastatin and the manner in which it should be provided locally. Within this context, the objective of this study was to evaluate the effect of a local application of simvastatin in the gel state on the repair of bone defects in rabbit femurs.

\section{Methods}

The experimental protocol (\#1913/08) was approved by the Ethics Committee of the Sao Paulo Federal University (UNIFESP). The study was designed as a randomized, controlled trial with a blinded assessment of the outcome.

Eighteen albino rabbits (Oryctolagus cuniculus), which were male, five months old, and weighed $3000 \mathrm{~g}$, underwent anesthesia with $0.1 \mathrm{ml}$ of xylazine $/ \mathrm{kg}$ and $50 \mathrm{mg} / \mathrm{kg}$ of ketamine injected intramuscularly. A linear $5 \mathrm{~cm}$ incision was made in the outer left thigh over the femoral epiphysis toward to the caudal region. With a digital electric micro-drill, which was under continuous saline irrigation and near the epiphysis, two standard and non-critical (4 $\mathrm{mm}$ diameter and $6 \mathrm{~mm}$ deep) bone cavities (proximal and distal) were prepared with a $10 \mathrm{~mm}$ distance between the two cavities. Subsequently, $1 \mathrm{mg}$ of simvastatin gel $(0.5 \mathrm{ml})$ mixed with a vehicle of $1 \%$ hydroxypropyl methylcellulose $(0.5$ $\mathrm{ml}$ ) was applied. To avoid dispersion of the simvastatin gel and to maintain the gel within the bone cavity, we used an absorbable, hydrolyzed and lyophilized collagen sponge (Technew-Cod. ANVISA $n^{\circ}$ : 80015520006) as a plug. To prevent invagination of non-osteogenic cells in both bone cavities, the cavities were coated with a membrane of resorbable bovine bone collagen (GenDermBaumer-Genius Biomaterials - Cod. ANVISA n ${ }^{\circ}$ : 10345500069).

The animals were randomly assigned to one of two groups. In the simvastatin group (SIN), the bone cavity was filled with an absorbable collagen sponge $(0.02 \mathrm{~g})$ soaked in 0.5 $\mathrm{ml}$ of simvastatin gel (SIN $1 \mathrm{mg} /$ HPMC $0.5 \mathrm{ml}$ ) and biological membrane collagen. In the control group $(\mathrm{CON})$, the bone cavity was filled with a blood clot and biological membrane collagen. The wound was sutured with mononylon 4.0. To promote analgesia and antimicrobial therapy, the animals received ketoprofen and enrofloxacin for five consecutive days. After surgery, the animals were randomly assigned into one of three periods of observation: seven, 21 or 42 days.

After euthanasia under anesthesia, the left femurs were fixed in 10\% formalin and were decalcified in trichloroacetic acid (5\%) for 20 days. Subsequently, each bone was sectioned level with the cavity and soaked in paraffin blocks. Finally, four semi-serial sections of each block were obtained, and each section was stained with hematoxylin and eosin (HE). The histological analysis of the slides from each group was performed using light microscopy (Axiophot 2, Carl Zeiss Brazil - Sao Paulo-SP). With the help of Adobe Photoshop for Windows 1997 (Microsoft - USA), the images were transferred to the program ImageJ 1.31p (National Institute of Health - USA) and evaluated blindly by the histologist. The areas of the bone cavities in each group and sub-group were analyzed by scoring and observing the following: vascularity, fibrosis, reactive bone formation, osteoblasts and osteoclasts.

For the immunohistochemical study, the sections were placed onto slides previously treated with a solution of organosilane (3-aminopropyltriethoxysilane, SIGMA, St. Louis, MO, USA, cat. 3648) and 20\% acetone. Then, the sections were placed in an oven maintained at $110^{\circ} \mathrm{C}$, subjected to deparaffinization in xylene, rehydrated in ethanol and washed in distilled water. The histological sections were then incubated with primary antibodies diluted in bovine serum albumin (1\% BSA in 
PBS). The antibodies were mouse monoclonal anti-osteocalcin to OCG4 (Abcam ab1342) and mouse monoclonal anti-VEGF JH121 (ab28775 Abcam). The slides were analyzed by scoring the immunoreactivity of the following markers: mouse monoclonal OCG4 primary antibody for osteocalcin (ab13421) and mouse monoclonal primary antibody JH121 to VEGF (ab28775).

\section{Statistical analysis}

To determine the differences between the two groups and between the three observation time periods, the results of the descriptive analysis were subject to a nonparametric Wilcoxon test (for paired samples), and the level for the rejection of the null hypothesis was set at $5 \%(\mathrm{p}<0.05)$.

\section{Results}

\section{Histological evaluation}

At seven days of observation (Figure 1), the semiquantitative assessment scores by frequency showed a significant presence of neo-vascularization in animals subject to local treatment with simvastatin. Other parameters, such as fibrosis, osteoblasts and osteoclasts, resulted in scores greater in the presence of simvastatin but did not achieve statistical significance. Osseous neoformation, as expected in this post-surgical time period, was not present in either group. At twenty-one days of observation (Figure 2), vascularization was significantly greater in the simvastatin group, and simultaneously, there was a greater presence of fibrosis and new bone formation in the simvastatin group. The scores for the presence of osteoblasts and osteoclasts, although greater in the simvastatin group, were not statistically significant. At forty-two days of observation (Figure 3), the scores for the vascularization and fibrosis did not show significant differences, but simvastatin promoted statistically significant differences in the score on bone formation and the presence of osteoblasts and osteoclasts.

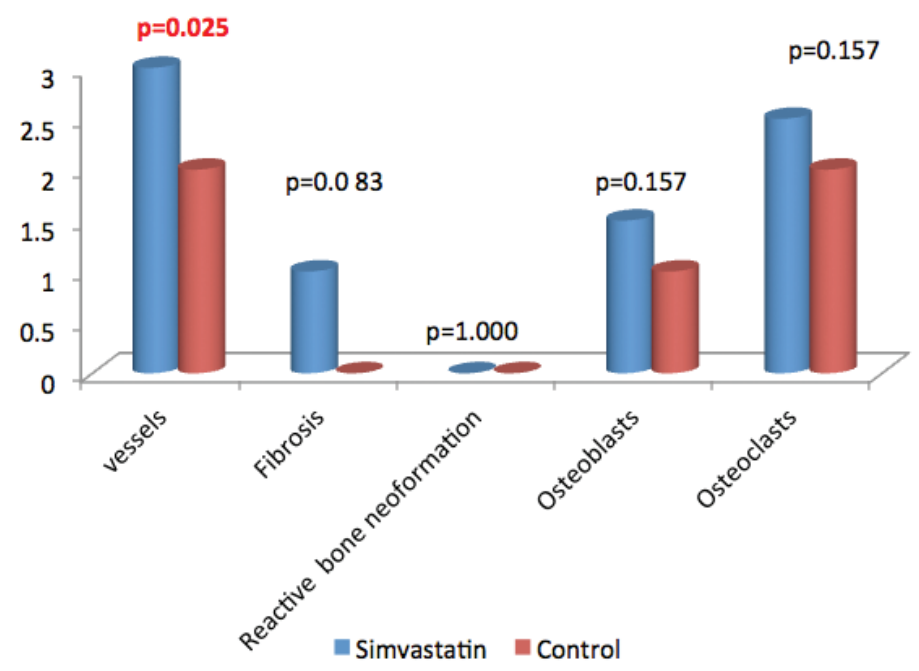

FIGURE 1 - Histological scores for vascularization, fibrosis, bone neoformation, osteoblasts and osteoclasts for the simvastatin and the control groups at seven days of observation (Wilcoxon test - $\mathrm{p}<0.05$ ).

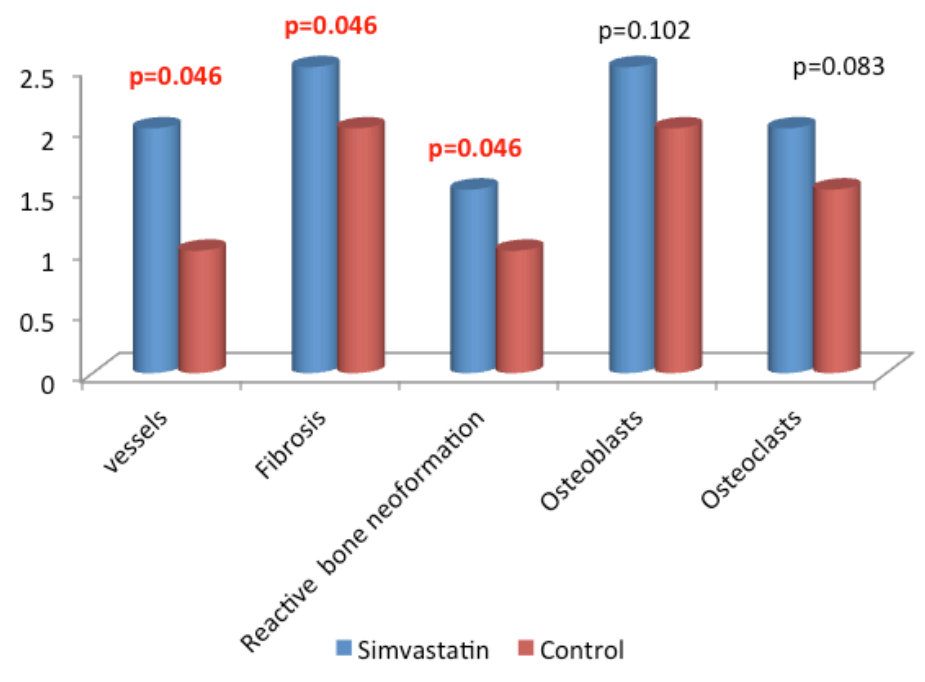

FIGURE 2 - Histological scores for vascularization, fibrosis, bone neoformation, osteoblasts and osteoclasts for the simvastatin and the control group at 21 days of observation (Wilcoxon test $-\mathrm{p}<0.05$ ).

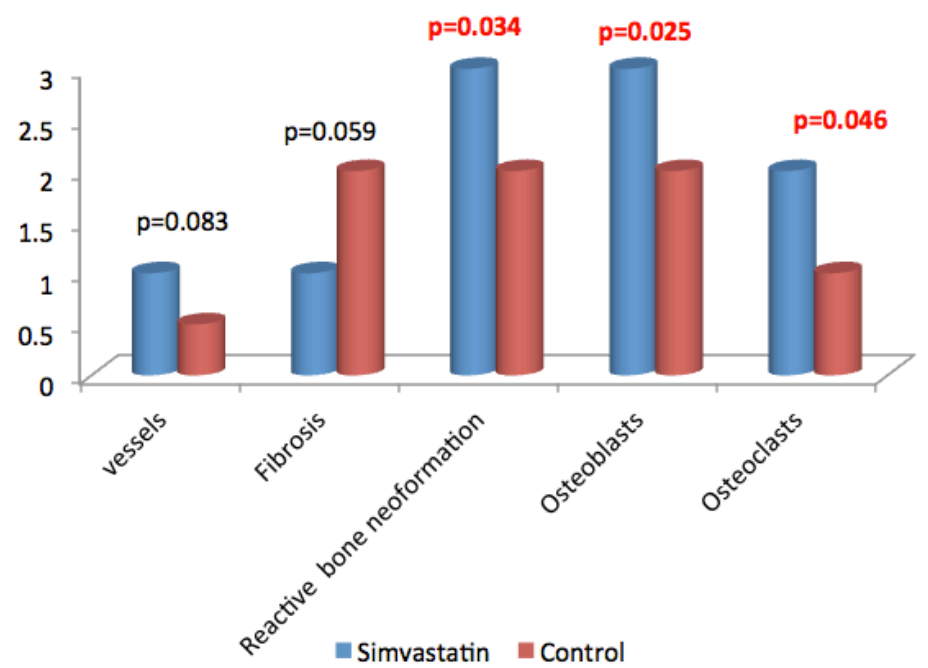

FIGURE 3 - Histological scores for vascularization, fibrosis, bone neoformation, osteoblasts and osteoclasts for the simvastatin and the control groups at 42 days of observation (Wilcoxon test $-\mathrm{p}<0.05$ ). 


\section{Immunohistochemistry evaluation}

At seven days of observation, there was a significant presence of VEGF, which matched the greater frequency of neovascularization observed from the HE staining. At twenty-one days, the VEGF was not statistically significant, which was unlike the osteocalcin, which was significantly more prevalent at this time. At forty-two days, the two markers did not show differences between the groups (Figure 4).

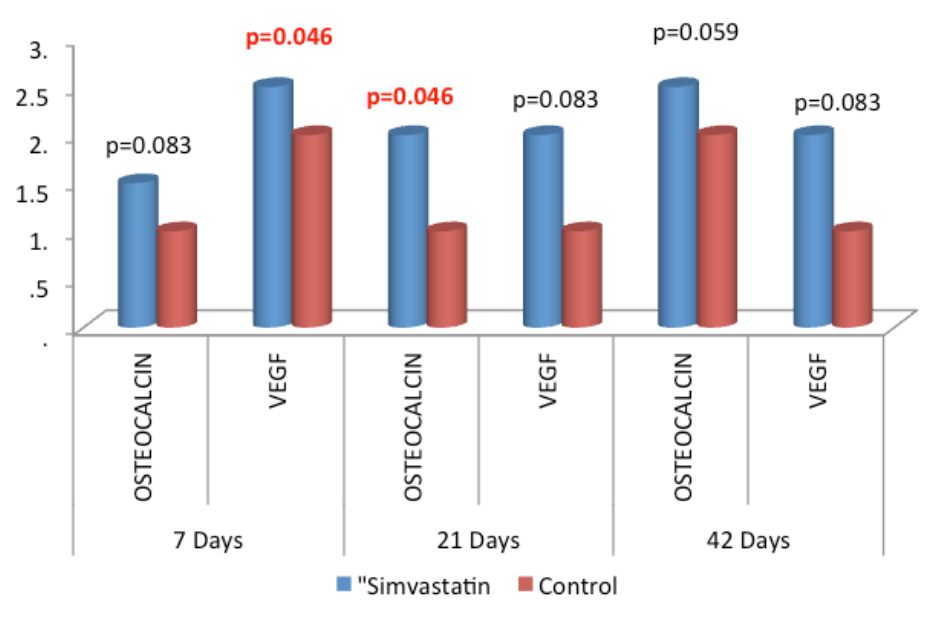

FIGURE 4 - Immunohistochemical scores for VEGF and osteocalcin for the simvastatin and the control group at 21 days of observation (Wilcoxon test $-\mathrm{p}<0.05)$.

\section{Discussion}

The development of new biomaterials has been conducted with the aim of identifying new methods of stimulating cytokines and growth factors focused on osteoinductive, osteoconductive and angiogenic actions. These biomaterials need to be easy to manipulate, be absorbable and have a physical structure capable of maintaining a space for bone formation without generating an appreciable immune response. Despite major advances in science and technology, there is not a substance that meets the majority of these requirements ${ }^{13,14}$.

Bone defects have been used as experimental models for the study of bone regeneration because the repair of these defects resembles the direct repair of fractures. Other models have been reported using animals, such as rats, rabbits, cats, dogs, pigs, monkeys, and have been conducted using bone defects in the calvaria, the jaw or in long bones ${ }^{15}$.

The rabbit model has the advantage that it is easy to handle the animals and allows for larger bone defects compared with rats, mice or guinea pigs. The exclusive use of males avoided the possible hormonal changes present in females because this could compromise the results. There is a consensus that the cycle of rabbit bone repair is completed in approximately 42 days ${ }^{16}$. Therefore, the evaluation carried out in periods of seven, 21 and 42 days allowed for the analysis of the initial, intermediate and final stages of bone repair ${ }^{15,16}$.

Our animal model created a bone defect that was regarded as a non-critical injury (4 $\mathrm{mm}$ diameter and $6 \mathrm{~mm}$ deep) and only removed the cortical layer. It was a defect that the body would be able to spontaneously repair by replacing the defect with new bone tissue without the need for some type of treatment. The purpose was to verify that the use of an osteoinductive material could shorten the time needed to repair the defect.

The femoral epiphysis has characteristics suitable for the preparation of cavities because it presents red bone marrow with a large amount of spongy bone and osteoprogenitor cells. Furthermore, the epiphysis of the femur allows for the creation of a defect with a larger diameter and depth without increasing the risk of fracture. With a trephine having a $4.0 \mathrm{~mm}$ diameter, it was possible to prepare two cavities in each femoral epiphysis with a minimal risk of hip fracture, which, in fact, did not occur in any animal.

Simvastatin is characterized as a lipophilic statin that can passively penetrate the cell membrane and exert its effect at the tissue level. We applied the simvastatin in gel form through an association with a vehicle: hidroxipropilenometilcelulose (HPMC), which is biocompatible, stable and able to slowly release simvastatin in its action site. The choice of the concentration of simvastatin (1.0 mg / ml gel 0.5 HPMC) was based on other reports that used similar doses: $2.2 \mathrm{mg}^{17}, 0.5 \mathrm{mg}^{11,18}, 0.1 \mathrm{mg} / 0.5 \mathrm{mg} / 1.0$ $\mathrm{mg} / 1.5 \mathrm{mg} / 2.2 \mathrm{mg}^{19}$. There are reports that the local use of highdose simvastatin impaired bone healing, which suggests that there might have been a stimulant effect on osteoclastic activity, which would result in the resorption of new bone formations ${ }^{9}$.

The success of bone repair depends on the permanence time and on the absorption of the material at the site of the bone defect ${ }^{20,21}$. The absorbable collagen sponge allowed the simvastatin gel to remain in the bone cavity, while not interfering with the drug's action ${ }^{11,18}$. A physical barrier (osteo promoter) was positioned and was composed of a resorbable collagen membrane overlying the opening of the bone defect with the intent of retaining the soft tissue and, thus, allowing only the cells originating from the bone tissue to occupy the area of the lesion.

Some authors did not observe new bone formation induced by simvastatin ${ }^{22-24}$. Other reports indicate that $\operatorname{simvastatin}^{17,25-28}$ influenced and stimulated new bone formation. There are few consistent studies on the anabolic effects and bioavailability of simvastatin, which is a fact that leads to such varying results among several studies ${ }^{29}$. 
The results obtained in this study for the period of seven days, which is considered to be the initial phase of bone healing, showed increased vascularity with a significantly greater amount of newly formed vessels in the simvastatin group compared with the control group (Figure 1). There was also a statistically significant greater antibody immunostaining for VEGF in the simvastatin group compared with the control group (Figure 4). These results are in agreement with other studies ${ }^{11,12,18}$, and it can be inferred that, in the acute phase of bone repair, simvastatin had a beneficial effect on stimulating the production of new vessels, which might reflect an advantage for subsequent stages of bone regeneration.

For the other variables, even with greater mean and median values in the simvastatin group, no significant difference was observed between the groups. It is possible that further study with a larger sample or a different marker of greater sensitivity can demonstrate the trend observed in these favorable results with the use of simvastatin. Slight fibrosis and no new bone formation was observed, which is to be expected at this stage. The moderate immunostaining for osteocalcin is also expected as there has not been sufficient stimuli for osteocalcin's manifestation, which should occur in the later stages of the process. The large amount of osteoclasts and moderate amounts osteoblasts were consistent with other reports in the literature ${ }^{11,12,18}$, which demonstrates the need for removing the remaining bone debris before the proliferation of osteoblasts and deposition of bone matrix, which is driven by osteocalcin.

After twenty-one days, we observed an intense fibrosis, and there was increased formation of dense connective tissue rich in collagen fibers in the simvastatin group compared with the control group. There was also moderate vascularization and early bone formation with greater scores in the simvastatin group compared with the control group (Figure 4). Greater expression for the osteocalcin antibody in the simvastatin group was found compared with the control group. There are reports that coincide with these data ${ }^{30,31}$. Additionally, at twenty-one days in the intermediate phase of bone regeneration, simvastatin showed favorable effects by stimulating a greater presence of connective tissue and vessels as well as a greater stimulation of the bone matrix by osteocalcin.

For the other variables, although the mean was identical and the median was greater in the simvastatin group, there was no statistically significant difference between the groups with slight immune-staining for VEGF, large amounts of osteoblasts and moderate amounts of osteoclasts. Again, a larger sample size or a more sensitive marker may help to confirm this favorable trend for the use of simvastatin in future studies.
During the forty-two day observation period, we documented an intense reactive bone formation as well as greater scores for bone tissue formation in the simvastatin group compared with the control group (Figure 3). More osteoblasts were observed with greater scores in the simvastatin group compared with the control group (Figure 3). The same occurred with the osteoclasts with greater scores in the simvastatin group compared with the control group. These results are in agreement with other studies ${ }^{16-19,26}$. In the late period of regeneration, simvastatin was able to stimulate a greater presence of osteoblasts and subsequent bone formation.

The remaining variables showed no statistically significant difference between the groups, but we observed discrete neovascularization in the two groups and moderate fibrosis still present in the control group. Intense and greater immune-staining for osteocalcin antibody in the simvastatin group was observed compared with the control group as well as mild immune-staining for VEGF in both groups (Figure 4).

Given the results, the proposed model of topical application of the simvastatin proved to be efficient and effective. The applied drug was stable and persisted in cavities that were made in the femurs of rabbits, which shows that this method of offering the drug should be studied in more detail in the future. Moreover, the beneficial effects of simvastatin have been shown at different stages during the biological process of bone repair. At the $7^{\text {th }}$ day, simvastatin's effect was greatly expressed in stimulating vascularization, and this time point is the crucial point of the acute phase. Fibroblast proliferation and marked bone formation was observed on the $21^{\text {th }}$ day and persisted to the $42^{\text {nd }}$ day with a significant expression of osteoblasts and osteoclasts.

\section{Conclusion}

Surgical defects created in rabbit femurs were treated locally with simvastatin gel to stimulate bone repair, which promoted an ameliorative effect in the morphological and immunohistochemical markers of bone regeneration.

\section{References}

1. Hollinger JO, Kleinschmidt JC. The critical size defect as an experimental model to test bone repair materials. J Craniofac Surg. 1990;1:60-8.

2. Mish CE, Dietsh F. Bone grafting materials in implant dentistry. Implant Dent. 1993;2:158-67.

3. Urist M. Bone: formation by autoinduction. Science. 1965;150(3698):893-9.

4. Yamaguchi A, Katagir T, Ikeda T, Wozney JM, V Rosen V, Wang EA, Kahn AJ, Suda T, Yoshiki S. Recombinant human bone morphogenetic protein-2 stimulates osteoblastic maturation and inhibits myogenic 
differentiation in vitro. J Cell Biol. 1991;113:681-7.

5. Tsumaki N, Yoshikawa $\mathrm{H}$. The role of bone morphogenetic proteins in endochondral bone formation. Cytokine Growth Factor Rev. 2005;16(3):279-85.

6. Yamashita M, Otsuka F, Mukai T, Yamanaka R, Otani H, Matsumoto Y, Nakamura E, Takano M, Sada KE, Makino H. Simvastatin inhibits osteoclast differentiation induced by bone morphogenetic protein-2 and RANKL through regulating MAPK, AKT and Src signaling. Regul Pept. 2010;162(1-3):99-108.

7. Mori M, Nishikawa T, Masuno K, Okamura T, Tanaka A, Shikimori M. Statins: candidates for promoting bone formation via BMP2. Oral Med Pathol. 2010;14(3):81-7.

8. Zhang Y, Zhang R, Li Y, He G, Zhang D, Zhang F. Simvastatin augments the efficacy of therapeutic angiogenesis induced by bone marrow-derived mesenchymal stem cells in a murine model of hindlimb ischemia. Mol Biol Rep. 2012;39(1):285-93.

9. Maritz FJ, Conradie MM, Hulley PA, Gopa LR, Hough S. Effect of statins on bone mineral density and bone histomorphometry in rodents. Arterioscler Thromb Vasc Biol. 2001;21(10):1636-41.

10. Sharif PS, Abdollahi M. A Systematic review on the relation between use of statins and osteoporosis. Int J Pharmacol. 2011;7(2):180-8. Available from http://scialert.net/abstract/?doi=ijp.2011.180.188 8.RL:

11. Wong RWK, Rabie ABM. Statin collagen grafts used to repair defects in the parietal bone of rabbits. J Oral Maxillofac Surg. 2003;41:244-8.

12. Oliveira NM, Correa VB, Chavez VEA. Early alveolar bone regeneration in rats after topical administration of simvastatin. Oral Surg Oral Med Oral Pathol Oral Radiol Endod. 2011;112(2):170-9.

13. Rimondini L, Nicoli-Aldini N, Fini M, Guzzardella G, Tschon M, Giardino R. In vivo experimental study on bone regeneration in critical bone defects using an injectable biodegradable PLA/PGA copolymer. Oral Surg Oral Med Oral Pathol Oral Radiol Endod. 2005;99(2):148-54.

14. Nagao H, Tachikawa N, Miki T, Oda M, Mori M, Takahashi K, Enomoto S. Effect of recombinant human bone morphogenetic protein- 2 on bone formation in alveolar ridge defects in dogs. Int $\mathrm{J}$ Oral Maxillofac Surg. 2002;31(1):66-72.

15. Schmitz JP, Hollinger JO. The critical size defect as an experimental model for craniomandibulofacial nonunions. Clin Orthop Relat Res. 1986;205:298-307.

16. MacNeill SR, Cobb CM, Rapley JW, Glaros AG, Spencer P. In vivo comparison of synthetic osseous graft materials. A preliminary study. J Clin Periodontol. 1999;26(4):239-45.

17. Thylin MR, Mcconnell JC, Schmid MJ, Reckling RR, Ojha J, Bhattacharyya I, Marx DB, Reinhardt RA. Effects of Simvastatin gels on murine calvarial bone. J Periodontol. 2002;73(10):1141-8.

18. Ozeç I, Killiç E, Gumus C, Goze F. Effect of local sinvastatin application on mandibular defects. J Craniofac Surg. 2007;18(3):546-50.

19. Stein D, Lee Y, Schmid MJ, Killpack B, Genrich MA, Narayana N, Marx DB, Cullen DM, Reinhardt RA. Local sinvastatin effects on mandibular bone growth and inflammation. J Periodontol. 2005;76(11):1861-70.

20. Nakashima M, Reddi A. H.The application of bone morphogenetic proteins to dental tissue engineering. Nat Biotechnol. 2003;21(9):1025-32.

21. Lutolf, MP,Lauer-Fields JL, Schmoekel HG. Metters AT, Weber FE, Fields GB, Hubbell JA. Synthetic matrix metalloproteinase-sensitive hydrogels for the conduction of tissue regeneration: engineering cellinvasion characteristics. Proc Natl Acad Sci. 2003;100(9):5413-8.

22. von Stechow D, Fish S, Yahalom D, Bab I, Chorev M, Müller R, Alexander JM. Does simvastatin stimulate bone formation in vivo? BMC Musculoskelet Disord. 2003; 4:8.
23. Sonobe M, Hattori K, Tomita N, Yoshikawa T, Aoki H, Takakura Y, Suguro T. Stimulatory effects of statins on bone marrow-derived mesenchymal stem cells. Study of a new therapeutic agent for fracture. Biomed Mater Eng. 2005;15(4):261-7.

24. Lima CE, Calixto JC, Anbinder AL. Influence of the association between simvastatin and demineralized bovine bone matrix on bone repair in rats. Braz Oral Res. 2011;25(1):42-8.

25. Wong RWK, Rabie ABM. Early healing pattern of statin-induced osteogenesis. Br J Oral Maxillofac Surg. 2005;43:46-50.

26. Mundy G, Garrett R, Harris S, Chan J, Chen D, Rossini G, Boyce B, Zhao M, Gutierrez G. Stimulation of bone formation in vitro and in rodents by statins. Science.1999;286:1946-9.

27. Karanxha L, Park SJ, Son WJ, Nör JE, Min KS. Combined effects of simvastatin and enamel matrix derivative on odontoblastic differentiation of human dental pulp cells. J Endod. 2013;39(1):76-82.

28. Chuang SC, Liao HJ, Li CJ, Wang GJ, Chang JK, Ho ML. Simvastatin enhances human osteoblast proliferation involved in mitochondrial energy generation. Eur J Pharmacol. 2013;714:74-82.

29. Mah J, Hung J, Wang J, Salih E. The efficacy of various alloplastic bone grafts on the healing of rat calvarial defects. Eur J Orthod. 2004;26(5):475-82.

30. Song C, Guo Z, Ma Q, Chen Z, Liu Z, Jia H, Dang G. Simvastatin induces osteoblastic differentiation e and inhibits adipocytic differentiation in mouse bone marrow stromal cells. Biochem Biophys Res Commun. 2003;308:458-62.

31. Liu M, Wang K, Tang T, Dai K, Zhu Z. The effect of simvastatin on the differentiation of marrow stromal cells from aging rats. Pharmazie. 2009;64(1):43-8.

\section{Correspondence:}

José Eduardo Gomes Cardim Rosselli

Rua Alexandre Levi, 100/31

015020-000 Sao Paulo - SP Brasil

Tel.: (55 11)2856-4778/99449-6008

jegcr@uol.com.br

Received: Oct 15, 2013

Review: Dec 16, 2013

Accepted: Jan 20, 2014

Conflict of interest: none

Financial source: Sao Paulo Research Foundation(FAPESP-2011/19237-5)

${ }^{1}$ Research performed at Operative Technique and Experimental Surgery Division, Department of Surgery, Sao Paulo Federal University (UNIFESP), Sao Paulo-SP, Brazil. Part of PhD degree thesis, Postgraduate Program in Interdisciplinary Surgical Science. Tutor: Murched Omar Taha. 